\title{
EXISTENCE OF THREE SOLUTIONS FOR A QUASILINEAR ELLIPTIC EQUATION INVOLVING THE $p(x)$-LAPLACE OPERATOR
}

\author{
RABIL AYAZOGLU (MASHIYEV) AND MUSTAFA AVCI
}

\begin{abstract}
In this paper, some existence results are obtained by using a three critical point theorem based on variational principle. In that context, we verify that a quasilinear elliptic equation involving the $p(x)$-Laplace operator has at least three weak solutions under Neumann boundary condition.
\end{abstract}

\section{INTRODUCTION AND PRELIMINARIES}

In the present paper, we study the existence of solutions of the $p(x)$ Laplacian Neumann problem

$$
\left\{\begin{array}{c}
-\operatorname{div}\left(|\nabla u|^{p(x)-2} \nabla u\right)+|u|^{p(x)-2} u=f(x, u)+\lambda g(x, u) \text { in } \Omega, \\
\frac{\partial u}{\partial \nu}=0 \text { on } \partial \Omega,
\end{array}\right.
$$

where $\Omega \subset \mathbb{R}^{N}(2 \leq p(x)<N)$ is a bounded domain with smooth boundary $\partial \Omega, \lambda \in \mathbb{R}, \nu$ is the outward unit normal to $\partial \Omega$ and $f, g: \Omega \times \mathbb{R} \rightarrow \mathbb{R}$ are Carathéodory functions which satisfy some given conditions.

The main argument used here is a three critical point theorem due to Bonanno [1]. However, this type of results were initially introduced by Ricceri (see $[13-15])$. On the other hand, Neumann problems of $\left(P_{\lambda}\right)$-type have been broadly investigated in recent years by many authors considering different conditions and using various methods. For example, in [12,18], the authors studied the $p(x)$-Laplacian Neumann problems of the following type

$$
\left\{\begin{array}{c}
-\operatorname{div}\left(|\nabla u|^{p(x)-2} \nabla u\right)+|u|^{p(x)-2} u=\lambda f(x, u) \text { in } \Omega, \\
\frac{\partial u}{\partial \nu}=0 \text { on } \partial \Omega,
\end{array}\right.
$$

2010 Mathematics Subject Classification. 35J60, 35B30, 35B40.

Key words and phrases. Variable exponent Lebesgue and Sobolev spaces, $p(x)$ Laplacian, Neumann problem, variational approach, three solutions. 
where $\Omega \in \mathbb{R}^{N}(N \geq 3)$ is a bounded domain with a smooth boundary, $\lambda>0$ is a real number, $p$ is a continuous function on $\Omega$ with $i n f_{y \in \bar{\Omega}} p(y)>N$ and $f: \Omega \times \mathbb{R} \rightarrow \mathbb{R}$ is a continuous function, $\nu$ the outward unit normal to $\partial \Omega$.

In [10], the author considered the following $p(x)$-Laplacian equations under the different kinds of boundary conditions

$$
\left\{\begin{array}{c}
-\operatorname{div}\left(|\nabla u|^{p(x)-2} \nabla u\right)+|u|^{p(x)-2} u=\lambda((f(x, u)+\mu g(x, u)) \text { in } \Omega, \\
\frac{\partial u}{\partial \nu}=\text { on } \partial \Omega,
\end{array}\right.
$$

where $\Omega \subset \mathbb{R}^{N}(N \geq 2)$ is a bounded domain with smooth boundary, $\mu, \lambda$ are constants and $\lambda>0$ and $\nu$ is the outward unit normal to $\partial \Omega$.

Moreover, in [17], the authors studied problem $\left(P_{\lambda}\right)$ for the case $p(x)=$ $p=$ const and consider the Dirichlet boundary conditions where the nonlinearities $f$ and $g$ obey some different conditions.

The $p(x)$-Laplace operator $-\Delta_{p(x)} u:=\operatorname{div}\left(|\nabla u|^{p(x)-2} \nabla u\right)$ is a natural generalization of the $p$-Laplace operator $-\Delta_{p} u:=-\operatorname{div}\left(|\nabla u|^{p-2} \nabla u\right)$ where $p>1$ is a real constant. The main difference between them is that the $p$-Laplacian operator is $(p-1)$-homogenous, that is, $\Delta_{p}(\mu u)=\mu^{p-1} \Delta_{p} u$ for every $\mu>0$, but the $p(x)$-Laplacian operator, when $p(x)$ is not constant, is not homogeneous. This causes many problems, some classical theories and methods, such as the Lagrange multiplier theorem and the theory of Sobolev spaces, are not applicable.

The study of differential equations and variational problems involving $p(x)$-growth conditions, has attracted a special interest because of the fact that there are some physical phenomena which can be modelled by such kind of equations, such as elastic mechanics, electrorheological fluids (sometimes referred to as 'smart fluids'), image processing. For more information we refer to $[2,3,7,9,11,16,19-21]$. In that context, it is accepted that the most convenient spaces for the mathematical modelling of such physical problems are variable exponent Lebesgue and Sobolev spaces.

In the sequel, we recall some basic properties of the variable exponent Lebesgue $L^{p(x)}(\Omega)$ and Sobolev spaces $W^{1, p(x)}$, where $\Omega \subset \mathbb{R}^{N}$ is a bounded domain. In that context, we refer to $[5,8]$ for further reading.

Set $C_{+}(\bar{\Omega})=\{h: h \in C(\bar{\Omega}), h(x)>1\}$ for all $x \in \bar{\Omega}$, and define

$$
h^{-}=\min _{x \in \bar{\Omega}} h(x) \text { and } h^{+}=\max _{x \in \bar{\Omega}} h(x), \forall h \in C_{+}(\bar{\Omega}) .
$$

For any $p \in C_{+}(\bar{\Omega})$, we define the variable exponent Lebesgue space $L^{p(x)}(\Omega)$ by

$$
L^{p(x)}(\Omega)=\left\{u: \Omega \rightarrow \mathbb{R} \text { is measurable: } \int_{\Omega}|u(x)|^{p(x)} d x<\infty\right\},
$$


The modular of $L^{p(x)}(\Omega)$ which is the mapping $\rho: L^{p(x)}(\Omega) \rightarrow \mathbb{R}$ is defined by

$$
\rho(u)=\int_{\Omega}|u|^{p(x)} d x .
$$

We define a norm, the so-called Luxemburg norm, on $L^{p(x)}(\Omega)$ by the formula

$$
|u|_{p(x)}=\inf \left\{\eta>0: \int_{\Omega}\left|\frac{u(x)}{\eta}\right|^{p(x)} d x \leq 1\right\}
$$

and then $\left(L^{p(x)}(\Omega),|\cdot|_{p(x)}\right)$ becomes a Banach space.

Also define the variable exponent Sobolev space $W^{1, p(x)}(\Omega)$ by

$$
W^{1, p(x)}(\Omega)=\left\{u \in L^{p(x)}(\Omega):|\nabla u| \in L^{p(x)}(\Omega)\right\},
$$

with the norm

$$
\|u\|:=\|u\|_{1, p(x)}=|u|_{p(x)}+|\nabla u|_{p(x)} .
$$

Moreover, it is well known that if $1<p^{-} \leq p^{+}<\infty$, then spaces $L^{p(x)}(\Omega)$ and $W^{1, p(x)}(\Omega)$ are separable and reflexive Banach spaces (see, e.g., $[5,8])$.

We note that we can use the following equivalent norm on $W^{1, p(x)}(\Omega)$ :

$$
\|u\|=\inf \left\{\eta>0: \int_{\Omega}\left(\left|\frac{\nabla u(x)}{\eta}\right|^{p(x)}+\left|\frac{u(x)}{\eta}\right|^{p(x)}\right) d x \leq 1\right\} .
$$

The modular of $W^{1, p(x)}(\Omega)$ which is the mapping $\rho_{p(x)}: W^{1, p(x)}(\Omega) \rightarrow \mathbb{R}$ is defined by

$$
\rho_{p(x)}(u)=\int_{\Omega}\left(|\nabla u|^{p(x)}+|u|^{p(x)}\right) d x .
$$

Proposition 1.1. [5, 8] If $u, u_{n} \in L^{p(x)}(\Omega)(n=1,2, \ldots)$, we have

( i) $|u|_{p(x)}<1(=1 ;>1) \Leftrightarrow \rho(u)<1(=1 ;>1)$;

(ii) $|u|_{p(x)}>1 \Longrightarrow|u|_{p(x)}^{p^{-}} \leq \rho(u) \leq|u|_{p(x)}^{p^{+}} ;|u|_{p(x)}<1 \Longrightarrow|u|_{p(x)}^{p^{+}} \leq$ $\rho(u) \leq|u|_{p(x)}^{p^{-}} ;$

(iii) $\lim _{n \rightarrow \infty}\left|u_{n}\right|_{p(x)}=0 \Leftrightarrow \lim _{n \rightarrow \infty} \rho\left(u_{n}\right)=0 ; \lim _{n \rightarrow \infty}\left|u_{n}\right|_{p(x)}=\infty \Leftrightarrow$ $\lim _{n \rightarrow \infty} \rho\left(u_{n}\right)=\infty$.

Proposition 1.2. [5, 8] Let $u, u_{n} \in W^{1, p(x)}(\Omega)$.

(i) $\lim _{n \rightarrow \infty}\left\|u_{n}-u\right\|=0$;

(ii) $\lim _{n \rightarrow \infty} \rho_{p(x)}\left(u_{n}-u\right)=0$;

(iii) $u_{n} \rightarrow u$ in measure in $\Omega$ and $\lim _{n \rightarrow \infty} \rho_{p(x)}\left(u_{n}\right)=\rho_{p(x)}(u)$. 
Proposition 1.3. [5, 8] Let $u, u_{n} \in W^{1, p(x)}(\Omega)$.

(i) $\|u\|<1(=1 ;>1) \Leftrightarrow \rho_{p(x)}(u)<1(=1 ;>1)$;

(ii) $\|u\|>1 \Longrightarrow\|u\|^{p^{-}} \leq \rho_{p(x)}(u) \leq\|u\|^{p^{+}} ;\|u\|<1 \Longrightarrow\|u\|^{p^{+}} \leq$ $\rho_{p(x)}(u) \leq\|u\|^{p^{-}}$

(iii) $\lim _{n \rightarrow \infty}\left\|u_{n}\right\|=0 \Leftrightarrow \lim _{n \rightarrow \infty} \rho_{p(x)}\left(u_{n}\right)=0 ; \lim _{n \rightarrow \infty}\left\|u_{n}\right\|=\infty \Leftrightarrow$ $\lim _{n \rightarrow \infty} \rho_{p(x)}\left(u_{n}\right)=\infty$.

The main results of the present paper are based on the following theorem obtained by G. Bonanno in [1].

Theorem A. Let $X$ be a separable and reflexive real Banach space, and let $\Phi, \Psi: X \rightarrow \mathbb{R}$ be two continuously Gâteaux differentiable functionals. Assume that $\Phi$ is sequentially weakly lower semicontinuous and even, that $\Psi$ is sequentially weakly continuous and odd, and that, for some $a>0$ and for each $\lambda \in[-a, a]$, the functional $\Phi+\lambda \Psi$ satisfies the Palais-Smale condition and

$$
\lim _{\|x\| \rightarrow+\infty}(\Phi(x)+\lambda \Psi(x))=+\infty .
$$

Finally, assume that there exists $k>0$ such that

$$
\inf _{x \in X} \Phi(x)<\inf _{|\Psi(x)|<k} \Phi(x) .
$$

Then, for every $a>0$ there exists an open interval $\Lambda \subset[-a ; a]$ and $a$ positive $\sigma$ real number, such that, for each $\lambda \in \Lambda$, the equation

$$
\Phi^{\prime}(x)+\lambda \Psi^{\prime}(x)=0
$$

admits at least three solutions in $X$ whose norms are less than $\sigma$.

\section{MAin RESUlts}

In order to apply Theorem A, we define the functionals $\Phi, \Psi: W^{1, p(x)}(\Omega)$ $\rightarrow \mathbb{R}$ by

$$
\begin{gathered}
\Psi(u)=\int_{\Omega} \frac{1}{p(x)}\left(|\nabla u|^{p(x)}+|u|^{p(x)}\right) d x-\int_{\Omega} F(x, u) d x, \\
\Phi(u)=-\int_{\Omega} G(x, u) d x,
\end{gathered}
$$

where

$$
F(x, u)=\int_{0}^{u} f(x, t) d t \text { and } G(x, u)=\int_{0}^{u} g(x, t) d t .
$$

Then energy functional associated to the problem $\left(P_{\lambda}\right)$ is $J_{\lambda}(u)=\Psi(u)+$ $\lambda \Phi(u)$. Arguments similar to those used in the proof of Proposition 3.1 in 
[11] imply that $\Phi, \Psi \in C^{1}\left(W^{1, p(x)}(\Omega), \mathbb{R}\right)$ with the derivatives given by

$$
\begin{gathered}
\left\langle\Psi^{\prime}(u), \varphi\right\rangle=\int_{\Omega}|\nabla u|^{p(x)-2} \nabla u \nabla \varphi d x+\int_{\Omega}|u|^{p(x)-2} u \varphi d x-\int_{\Omega} f(x, u) \varphi d x, \\
\left\langle\Phi^{\prime}(u), \varphi\right\rangle=-\int_{\Omega} g(x, u) \varphi d x
\end{gathered}
$$

for any $u, \varphi \in W^{1, p(x)}(\Omega)$.

Let $u \in W^{1, p(x)}(\Omega)$ is a weak solution of $\left(P_{\lambda}\right)$ if

$\int_{\Omega}|\nabla u|^{p(x)-2} \nabla u \nabla \varphi d x+\int_{\Omega}|u|^{p(x)-2} u \varphi d x-\int_{\Omega}[f(x, u)+\lambda g(x, u)] \varphi d x=0$

for every $\varphi \in W^{1, p(x)}(\Omega)$. So, the weak solution of the problem $\left(P_{\lambda}\right)$ are precisely critical points of the energy functional

$$
J_{\lambda}(u)=\int_{\Omega} \frac{1}{p(x)}\left(|\nabla u|^{p(x)}+|u|^{p(x)}\right) d x-\int_{\Omega} F(x, u) d x-\lambda \int_{\Omega} G(x, u) d x .
$$

Now, we state our main results.

Through the present paper, if not otherwise stated, we assume that $f, g: \bar{\Omega} \times \mathbb{R} \rightarrow \mathbb{R}$ are Carathéodory functions which satisfy the following conditions:

$\left(f_{1}\right)$ There exist constants $c_{1}, c_{2}>0$ such that

$$
|f(x, t)|,|g(x, t)| \leq c_{1}+c_{2}|t|^{q(x)-1} \text { for a.e. } x \in \bar{\Omega} \text { and for all } t \in \mathbb{R} \text {, }
$$

where $q \in C_{+}(\bar{\Omega})$ and $q(x)<p(x)<p^{*}(x)=\frac{N p(x)}{N-p(x)}$ for a.e. $x \in \bar{\Omega}$.

$\left(f_{2}\right) h, s \in C_{+}(\bar{\Omega})$ satisfies the following conditions

$$
\frac{1}{p(x)}+\frac{q(x)}{s(x)}=1 \text { for a.e. } x \in \bar{\Omega},
$$

and

$$
\frac{1}{p(x)}+\frac{p^{-}}{h(x)}=1 \text { for a.e. } x \in \bar{\Omega},
$$

where $s^{-} \in\left(p^{+}, h^{+}\right)$and $h(x)<p^{*}(x)$.

$\left(f_{3}\right)$ There exist $\theta>p^{+}$and $t_{*}>0$ such that

$$
|t| \geq t_{*} \Longrightarrow 0<\theta F(x, t) \leq t f(x, t) \text { for a.e. } x \in \bar{\Omega} \text {. }
$$

i) $f(x, t)=o\left(|t|^{p^{-}-1}\right)$ as $t \rightarrow 0$ uniformly for $x \in \bar{\Omega}$, 
ii) $g(x, t)=o\left(|t|^{p^{-}-1}\right)$ as $t \rightarrow 0$ uniformly for $x \in \bar{\Omega}$.

$\left(f_{5}\right) f(x, \cdot)$ is odd and $g(x, \cdot)$ is even for all $x \in \bar{\Omega}$.

Theorem 2.1. Let $f, g: \bar{\Omega} \times \mathbb{R} \rightarrow \mathbb{R}$ be two Carathéodory functions satisfying the conditions $\left(f_{1}\right)-\left(f_{5}\right)$. Then, for every $a>0$, there exists an open interval $\Lambda \subset[-a, a]$ and a positive real number $\sigma$, such that for every $\lambda \in \Lambda$, Neumann problem $\left(P_{\lambda}\right)$ admits at least three solutions whose norms are less than $\sigma$.

Proof. From $\left(f_{1}\right)$ and $\left(f_{4}\right)$, given $\varepsilon>0$ there exists a positive constant $C_{\varepsilon}$, independent of $t$, such that

$$
|F(x, t)|,|G(x, t)| \leq \varepsilon|t|^{p^{-}}+C_{\varepsilon}|t|^{q(x)} \text { for all }(x, t) \in \bar{\Omega} \times \mathbb{R} .
$$

So the functional $\Psi$ is continuously Gâteaux differentiable functional and sequentially weakly continuous in $W^{1, p(x)}(\Omega)$ (see [10]). Also, by $\left(f_{1}\right)$ we know the functional $\Phi$ is sequentially weakly continuous in $W^{1, p(x)}(\Omega)$ (see $[6])$.

Since $p(x)<p^{*}(x)$ and $q(x)<p^{*}(x)$, then $W^{1, p(x)}(\Omega) \hookrightarrow L^{q(x)}(\Omega)$ and $W^{1, p(x)}(\Omega) \hookrightarrow L^{p^{+}}(\Omega) \hookrightarrow L^{p^{-}}(\Omega)$ (see [4]), with a continuous and compact embedding, what implies the existence of $c_{3}, c_{4}>0$

$$
|u|_{q(x)} \leq c_{3}\|u\| \text { for all } u \in W^{1, p(x)}(\Omega) .
$$

and

$$
|u|_{p^{-}} \leq|u|_{p^{+}} \leq c_{4}\|u\| \text { for all } u \in W^{1, p(x)}(\Omega) .
$$

By Proposition 1.1, Proposition 1.3, (2.2) and (2.3) we deduce that

$$
\begin{aligned}
\int_{\Omega} G(x, u) d x & \leq \varepsilon \int_{\Omega}|u|^{p^{-}} d x+C_{\varepsilon} \int_{\Omega}|u|^{q(x)} d x \\
& \leq \varepsilon|u|_{L^{p^{-}}}^{p^{-}}+C_{\varepsilon} \max \left(|u|_{q(x)}^{q^{-}},|u|_{q(x)}^{q^{+}}\right) \\
& \leq \varepsilon c_{4}\|u\|^{p^{-}}+c_{3} C_{\varepsilon} \max \left\{\|u\|^{q^{-}},\|u\|^{q^{+}}\right\} .
\end{aligned}
$$

And similarly, we have

$$
\int_{\Omega} F(x, u) d x \leq \varepsilon c_{4}\|u\|^{p^{-}}+c_{3} C_{\varepsilon} \max \left\{\|u\|^{q^{-}},\|u\|^{q^{+}}\right\} .
$$


For $\lambda \in \mathbb{R}$, from $\|u\|>1$, we deduce that

$$
\begin{aligned}
J_{\lambda}(u)= & \Psi(u)+\lambda \Phi(u)=\int_{\Omega} \frac{1}{p(x)}\left(|\nabla u|^{p(x)}+|u|^{p(x)}\right) d x \\
& -\int_{\Omega} F(x, u) d x-\lambda \int_{\Omega} G(x, u) d x \\
\geq & \frac{1}{p^{+}}\|u\|^{p^{-}}-2 \varepsilon c_{4}\|u\|^{p^{-}}-2\left(c_{3} C_{\varepsilon}+|\lambda|\right)\|u\|^{q+} .
\end{aligned}
$$

Choosing $\varepsilon=\frac{1}{4 c_{4} p^{+}}$, we get

$$
J_{\lambda}(u) \geq \frac{1}{2 p^{+}}\|u\|^{p^{-}}-2\left(c_{3} C_{\varepsilon}+|\lambda|\right)\|u\|^{q+} .
$$

Since $p^{-}>q^{+}$, it follows that

$$
\Psi(u)+\lambda \Phi(u) \rightarrow \infty \text { as }\|u\| \rightarrow \infty,
$$

i.e., $\Psi(u)+\lambda \Phi(u)$ coercive on $W^{1, p(x)}(\Omega)$.

Now, we prove that $J_{\lambda}$ satisfies the Palais-Smale $(P S)$ condition. Suppose $\left\{u_{n}\right\}$ is a $(P S)$ sequence of $J_{\lambda}$, that is, there exists $C>0$ such that

$$
J_{\lambda}\left(u_{n}\right) \rightarrow C, J_{\lambda}^{\prime}\left(u_{n}\right) \rightarrow 0 \text { as } n \rightarrow \infty .
$$

Assume that $\left\|u_{n}\right\| \rightarrow \infty$ as $n \rightarrow \infty$. But this contradicts with $J_{\lambda}\left(u_{n}\right) \rightarrow C$ since (2.4) holds. Thus, $\left\{u_{n}\right\}$ must be bounded. We may assume that there exists $u_{0} \in W^{1, p(x)}(\Omega)$ satisfying

$$
\begin{aligned}
u_{n} \rightarrow u_{0} \text { in } W^{1, p(x)}(\Omega), u_{n} \rightarrow u_{0} \text { in } L^{q(x)}(\Omega) & (\text { by }(2.2)), \\
& u_{n}(x) \rightarrow u_{0}(x) \text { a.e. on } \Omega .
\end{aligned}
$$

Observe that

$$
\begin{aligned}
\left\langle J_{\lambda}^{\prime}\left(u_{n}\right), u_{n}-u_{0}\right\rangle=\int_{\Omega}\left(\left|\nabla u_{n}\right|^{p(x)-2} \nabla u_{n} \nabla\left(u_{n}-u_{0}\right)\right. & \left.-\left|u_{n}\right|^{p(x)-2} u_{n}\left(u_{n}-u_{0}\right)\right) d x \\
-\int_{\Omega} f\left(x, u_{n}\right)\left(u_{n}-u_{0}\right) d x & -\lambda \int_{\Omega} g\left(x, u_{n}\right)\left(u_{n}-u_{0}\right) d x .
\end{aligned}
$$

We already know that

$$
\left\langle J_{\lambda}^{\prime}\left(u_{n}\right), u_{n}-u_{0}\right\rangle \rightarrow 0 \text { as } n \rightarrow \infty .
$$

By $\left(f_{1}\right)$ we have

$$
\int_{\Omega} f\left(x, u_{n}\right)\left(u_{n}-u_{0}\right) d x \rightarrow 0 \text { as } n \rightarrow \infty
$$


and

$$
\int_{\Omega} g\left(x, u_{n}\right)\left(u_{n}-u_{0}\right) d x \rightarrow 0 \text { as } n \rightarrow \infty .
$$

Using this, (2.5) and (2.6) we obtain

$$
\int_{\Omega}\left(\left|\nabla u_{n}\right|^{p(x)-2} \nabla u_{n} \nabla\left(u_{n}-u_{0}\right)-\left|u_{n}\right|^{p(x)-2} u_{n}\left(u_{n}-u_{0}\right)\right) d x \rightarrow 0
$$

as $n \rightarrow \infty$. This together with the convergence of $u_{n} \rightarrow u_{0}$ in $W^{1, p(x)}(\Omega)$, implies that

$$
u_{n} \rightarrow u_{0} \text { in } W^{1, p(x)}(\Omega) \text { as } n \rightarrow \infty .
$$

Hence, $J_{\lambda}$ satisfies the $(P S)$ condition.

Next, we want to prove that

$$
\inf _{u \in W^{1, p(x)}(\Omega)} \Psi(u)<0 .
$$

From $\left(f_{3}\right)$ one easy deduces that

$$
F(x, t) \geq \frac{F\left(x, t_{*}\right)}{t_{*}^{\theta}} t^{\theta},
$$

for $x \in \bar{\Omega}$ and all $t \geq t_{*}$. Thus, there are $\epsilon>1$ and nonnegative $u \in$ $W_{0}^{1, p(x)}(\Omega)$ such that $\left\{x \in \bar{\Omega}: u(x) \geq t_{*}\right\}$, then we have

$$
\begin{aligned}
\int_{\Omega} F(x, \epsilon u) d x & \geq \int_{\left\{\epsilon u \geq t_{*}\right\}} F(x, \epsilon u) d x \geq \frac{\epsilon^{\theta}}{t_{*}^{\theta}} \int_{\left\{\epsilon u \geq t_{*}\right\}} F\left(x, t_{*}\right) u^{\theta} d x \\
& \geq \frac{\epsilon^{\theta}}{t_{*}^{\theta}} \int_{\left\{u \geq t_{*}\right\}} F\left(x, t_{*}\right) u^{\theta} d x \geq \epsilon^{\theta} \int_{\left\{u \geq t_{*}\right\}} F\left(x, t_{*}\right) d x>0
\end{aligned}
$$

(recall $F \geq 0$ and $F\left(., t_{*}\right)>0$ almost everywhere). Then by Proposition 1.3 for all $(x, t) \in \bar{\Omega} \times \mathbb{R}$, we have

$$
\begin{aligned}
\Psi(\epsilon u) & =\int_{\Omega} \frac{|\nabla(\epsilon u)|^{p(x)}+|\epsilon u|^{p(x)}}{p(x)} d x-\int_{\Omega} F(x, \epsilon u) d x \\
& \leq \int_{\Omega} \frac{|\nabla(\epsilon u)|^{p(x)}+|\epsilon u|^{p(x)}}{p(x)} d x-\int_{\Omega} F(x, \epsilon u) d x \\
& \leq \frac{\epsilon^{p^{+}}}{p^{-}}\|u\|^{p^{+}}-\epsilon^{\theta} \int_{\left\{u \geq t_{*}\right\}} F\left(x, t_{*}\right) d x \\
& \leq \frac{\epsilon^{p^{+}}}{p^{-}}\|u\|^{p^{+}}-\epsilon^{\theta} \int_{\left\{u \geq t_{*}\right\}} F\left(x, t_{*}\right) d x .
\end{aligned}
$$

From the assumption on $\theta$ (see $\left(f_{3}\right)$ ), it is obvious that $\theta>p^{+}$, so this implies $\Psi(\epsilon u) \rightarrow-\infty$ as $\epsilon \rightarrow \infty$. Thus (2.7) holds. 
When $\|u\|$ is small enough, by (2.1) we have

$$
\begin{aligned}
\Psi(u) & =\int_{\Omega} \frac{1}{p(x)}\left(|\nabla u|^{p(x)}+|u|^{p(x)}\right) d x-\varepsilon \int_{\Omega}|u|^{p^{-}} d x-C_{\varepsilon} \int_{\Omega}|u|^{q(x)} d x \\
& \geq \frac{1}{p^{+}}\|u\|^{p^{+}}-\varepsilon \int_{\Omega}|u|^{p^{-}} d x-C_{\varepsilon} \int_{\Omega}|u|^{q(x)} d x
\end{aligned}
$$

Considering $\left(f_{2}\right)$ and applying Young's inequality, we have

$$
|u|^{p^{-}} \leq \varepsilon c_{5}+C_{\varepsilon}|u|^{h(x)}
$$

and

$$
|u|^{q(x)} \leq \varepsilon c_{6}+\bar{C}_{\varepsilon}|u|^{s(x)} .
$$

Since $\|u\|<1$, we deduce

$$
\int_{\Omega}|u|^{q(x)} d x \leq \varepsilon c_{7}+\bar{C}_{\varepsilon} \int_{\Omega}|u|^{s(x)} d x \leq \varepsilon c_{7}+\widehat{C}_{\varepsilon}\|u\|^{s^{-}} .
$$

Replacing these in (2.8), it results that

$$
\begin{aligned}
\Psi(u) & \geq \frac{1}{p^{+}}\|u\|^{p^{+}}-c_{8}\|u\|^{s^{-}}-c_{9}\|u\|^{h^{-}}-\varepsilon c_{10} \\
& \geq \frac{1}{p^{+}}\|u\|^{p^{+}}-c_{11}\|u\|^{s^{-}}-\varepsilon c_{10} \\
& =\frac{1}{p^{+}}\|u\|^{p^{+}}\left(1-c_{11}\|u\|^{s^{-}-p^{+}}\right)-\varepsilon c_{10} .
\end{aligned}
$$

Set

$$
\eta=\inf \left(\frac{1}{2},\left(\frac{1}{2 c_{11}}\right)^{\frac{1}{s^{-}-p^{+}}}\right) .
$$

For $u \in W^{1, p(x)}(\Omega)$ with $\|u\|=\eta$, we have

$$
\Psi(u) \geq \frac{1}{2 p^{+}}\|u\|^{p^{+}}-\varepsilon c_{10} \geq \frac{1}{4 p^{+}} \eta^{p^{+}}-\varepsilon c_{10} .
$$

Choose $\varepsilon=\frac{\eta^{p^{+}}}{8 c_{10} p^{+}}$, then we have

$$
\Psi(u) \geq \frac{1}{8 p^{+}}\|u\|^{p^{+}}>0 .
$$

Hence, there exists $k>0$ such that

$$
\inf _{|\Phi(u)|<k} \Psi(u)=0 .
$$

So we have

$$
\inf _{u \in W^{1, p(x)}(\Omega)} \Psi(u)<\inf _{|\Phi(u)|<k} \Psi(u) .
$$

The condition $\left(f_{6}\right)$ implies $\Psi$ is even and $\Phi$ is odd. All the assumptions of Theorem A are verified. Thus, for every $a>0$ there exists an open interval 
$\Lambda \subset[-a, a]$ and a positive real number $\sigma$, such that for every $\lambda \in \Lambda$, problem $\left(P_{\lambda}\right)$ admits at least three weak solutions in $W^{1, p(x)}(\Omega)$ whose norms are less than $\sigma$.

Theorem 2.2. Suppose that $f$ and $g$ satisfy assumptions $\left(f_{1}\right)-\left(f_{3}\right)$, $\left(f_{4}\right)(i i),\left(f_{5}\right)$, and that there exists a nonempty open set $\Omega_{1} \subseteq \Omega$ such that $\left(f_{4}^{\prime}\right)$

$$
\liminf _{t \rightarrow 0} \frac{\int_{0}^{t} f(x, t) d t}{|t|^{p^{-}}}=+\infty \text { uniformly for } x \in \Omega_{1} .
$$

Then, for every $a>0$, there exists an open interval $\Lambda \subset[-a, a]$ and $a$ positive real number $\sigma$, such that for every $\lambda \in \Lambda$, Neumann problem $\left(P_{\lambda}\right)$ admits at least three solutions whose norms are less than $\sigma$.

Proof. The proof is similar to the Theorem 2.1. So we only give a sketch of it. By the Theorem 2.1, the functional $\Psi, \Phi$ are sequentially weakly lower semicontinuous and continuously Gâteaux differentiable in $W^{1, p(x)}(\Omega), \Psi$ is even and $\Phi$ is odd. For every $\lambda \in \mathbb{R}$, the functional $\Psi(u)+\lambda \Phi(u)$ satisfies the $(P S)$ condition and $(2.4)$.

From $\left(f_{4}^{\prime}\right)$, we can find $\delta>0$ such that for any $H>0$ one has

$$
\inf _{x \in \Omega_{1}} \int_{0}^{t} f(x, t) d t>H|t|^{p^{-}} \text {for every } 0<|t| \leq \delta .
$$

We choose a nonzero nonnegative function $v \in C_{0}^{\infty}(\Omega)$ with $\inf _{x \in \Omega_{1}} v(x)>$ 0 , put $H>\|v\|^{p^{-}} / p^{-} \int_{\Omega}|v|^{p^{-}} d x$. Take a $\varepsilon>0$ such that $\varepsilon \sup _{x \in \Omega_{1}} v(x)<$ $\delta$, and let $u_{0}=\varepsilon v$. Then, we obtain

$$
\begin{aligned}
\psi(\varepsilon v) & \leq \frac{1}{p^{-}}\|\varepsilon v\|^{p^{-}}-\int_{\Omega}\left(\int_{0}^{\varepsilon v} f(x, \eta) d \eta\right) d x \\
& \leq \frac{\varepsilon^{p^{-}}}{p^{-}}\|v\|^{p^{-}}-H \varepsilon^{p^{-}} \int_{\Omega}|v|^{p^{-}} d x<0 .
\end{aligned}
$$

So, we get

$$
\inf _{u \in W^{1, p(x)}(\Omega)} \Psi(u)<0 .
$$

By the proof of Theorem 2.1, we know that there exists $k>0$ such that

$$
\inf _{u \in W^{1, p(x)}(\Omega)} \Psi(u)<\inf _{|\Phi(u)|<k} \Psi(u) .
$$

According to the Theorem A, for every $a>0$, there exists an open interval $\Lambda \subset[-a, a]$ and a positive real number $\sigma$, such that for every $\lambda \in \Lambda$, problem $\left(P_{\lambda}\right)$ admits at least three solutions whose norms are less than $\sigma$. 
Theorem 2.3. Suppose that $f$ and $g$ satisfy assumptions $\left(f_{1}\right)-\left(f_{3}\right)$, $\left(f_{4}\right)(i i),\left(f_{5}\right)$, and that there exists a nonempty open set $\Omega_{1} \subseteq \Omega$ such that $\left(f_{4}^{\prime \prime}\right)$

$\liminf _{t \rightarrow 0} \frac{\int_{0}^{t} f(x, t) d t}{|t|^{p(x)}}>-\infty, \limsup _{t \rightarrow 0} \frac{\int_{0}^{t} f(x, t) d t}{|t|^{p^{-}}}=+\infty$ uniformly for $x \in \Omega_{1}$.

Then, for every $a>0$, there exists an open interval $\Lambda \subset[-a, a]$ and $a$ positive real number $\sigma$, such that for every $\lambda \in \Lambda$, Neumann problem $\left(P_{\lambda}\right)$ admits at least three solutions whose norms are less than $\sigma$.

Proof. The conclusion follows by applying both Theorems 2.1 and 2.2. Here we only prove

$$
\inf _{u \in W^{1, p(x)}(\Omega)} \Psi(u)<0 .
$$

From condition $\left(f_{4}^{\prime \prime}\right)$, there exist $L>0, \delta>0$ such that

$$
\inf _{x \in \Omega_{1}} \int_{0}^{t} f(x, t) d t>-L|t|^{p(x)} \geq-L|t|^{p^{-}} \text {for every } 0<t \leq \delta .
$$

Let us consider a compact set $\Omega_{0} \subset \Omega_{1}$, with $\left|\Omega_{0}\right|=(L+1)\left|\Omega_{1} / \Omega_{0}\right|$ and a nonzero nonnegative function $v \in C_{0}^{\infty}(\Omega)$ such that

$$
\begin{gathered}
v(x) \equiv 1 \text { if } x \in \Omega_{0}, \\
0<v(x) \leq 1 \text { if } x \in \Omega_{1} / \Omega_{0}, \\
v(x) \equiv 0 \text { if } x \in \Omega / \Omega_{1} .
\end{gathered}
$$

Then we have $|v| \leq 1$ and $\int_{\Omega_{1} / \Omega_{0}}|v|^{p^{-}} d x \leq\left|\Omega_{1} / \Omega_{0}\right|$. Thanks to the condition $\left(f_{4}^{\prime \prime}\right)$, there exists $t^{\prime} \in \mathbb{R}$ such that

$$
\inf _{x \in \Omega_{1}} \int_{0}^{t^{\prime}} f(x, t) d t \geq \max \left(1, \frac{\int_{\Omega}|v|^{p^{-}} d x}{\left|\Omega_{1} / \Omega_{0}\right|}\right)\left|t^{\prime}\right|^{p^{-}} \text {for every } 0<\left|t^{\prime}\right| \leq \delta .
$$

By (2.10) and (2.11), we get

$$
\int_{0}^{t^{\prime} v} f(x, t) d t \geq-L \inf _{x \in \Omega_{1}} \int_{0}^{t^{\prime}} f(x, t) d t \text { for every } 0<\left|t^{\prime}\right| \leq \delta .
$$


Taking into account (2.11) and (2.12), we have

$$
\begin{aligned}
\psi\left(t^{\prime} v\right) & \leq \frac{1}{p^{-}}\left\|t^{\prime} v\right\|^{p^{-}}-\int_{\Omega}\left(\int_{0}^{t^{\prime} v} f(x, t) d t\right) d x \\
& \leq \frac{\left|t^{\prime}\right|^{p^{-}}}{p^{-}}\|v\|^{p^{-}}-\left|\Omega_{0}\right| \inf _{x \in \Omega_{1}} \int_{0}^{t^{\prime}} f(x, t) d t-\left|\frac{\Omega_{1}}{\Omega_{0}}\right| \inf _{x \in \Omega_{1}} \int_{0}^{t^{\prime} v} f(x, t) d t \\
& \leq \frac{\left|t^{\prime}\right|^{p^{-}}}{p^{-}}\|v\|^{p^{-}}-(L+1)\left|\frac{\Omega_{1}}{\Omega_{0}}\right| \inf _{x \in \Omega_{1}} \int_{0}^{t^{\prime}} f(x, t) d t \\
& \leq \frac{\left|t^{\prime}\right|^{p^{-}}}{p^{-}}\|v\|^{p^{-}}-\left|\frac{\Omega_{1}}{\Omega_{0}}\right| \inf _{x \in \Omega_{1}} \int_{x \in \Omega_{1}}^{t_{0}} f(x, t) d t \\
& \leq \frac{\left|t^{\prime}\right|^{p^{-}}}{p^{-}}\|v\|^{p^{-}}-\left|t^{\prime}\right|^{p^{-}} \int_{\Omega}|v|^{p^{-}} d x \\
& \leq \frac{\left|t^{\prime}\right|^{p^{-}}}{p^{-}}\|v\|^{p^{-}}-\left|t^{\prime}\right|^{p^{-}}\|v\|^{p^{-}}<0,
\end{aligned}
$$

and so (2.9) holds. Arguing as in Theorem 2.2, we have the same results.

Remark 2.4. In particular, under the same assumptions, there exists a sequence $\left\{\lambda_{n}\right\}$ converging to 0 such that, for each $n \in \mathbb{N}$, the problem

$$
\begin{gathered}
-\operatorname{div}\left(|\nabla u|^{p(x)-2} \nabla u\right)+|u|^{p(x)-2} u=f(x, u)+\lambda_{n} g(x, u) \text { in } \Omega, \\
\frac{\partial u}{\partial \nu}=0 \quad \text { on } \partial \Omega,
\end{gathered}
$$

admits at least three weak solutions in $W^{1, p(x)}(\Omega)$.

\section{REFERENCES}

[1] G. Bonanno, Some remarks on a three critical points theorem, Nonlinear Anal., 54 (2003), 651-665.

[2] L. Diening, Theoretical and Numerical Results for Electrorheological Fluids, PhD, University of Frieburg, Germany, 2002.

[3] L. Diening, P. Harjulehto, P. Hästö, and M. Ruzicka, Lebesgue and Sobolev spaces with variable exponents, Lect. Notes Math., vol. 2017, Springer-Verlag, Berlin, 2011.

[4] X. L. Fan, J. S. Shen and D. Zhao, Sobolev embedding theorems for spaces $W^{k, p(x)}(\Omega)$, J. Math. Anal. Appl., 262 (2001), 749-760.

[5] X. L. Fan and D. Zhao, On the spaces $L^{p(x)}(\Omega)$ and $W^{m, p(x)}(\Omega)$, J. Math. Anal. Appl., 263 (2001), 424-446.

[6] X. L. Fan and S. G. Deng, Remarks on Ricci's variatonal principle and applications to the $p(x)$-Laplacian equations, Nonlinear Anal., 67 (2007), 3064-3075. 
[7] P. Hästö, The $p(x)$-Laplacian and applications, J. Anal. 15 (2007), 53-62 (Special proceedings volume).

[8] O. Kovăčik and J. Răkosnik, On spaces $L^{p(x)}$ and $W^{1, p(x)}$, Czech. Math. J., 41 (1991), $592-618$

[9] F. Li, Z. Li and Pi L. Ling, Variable exponent functionals in image restoration, Appl. Math. Comput., 216 (3) (2010), 870-882.

[10] Q. Liu, Existence of three solutions for $p(x)$-Laplacian equations, Nonlinear Anal., 68 (2008), 2119-2127.

[11] M. Mihăilescu and V. Rădulescu, A multiplicity result for a nonlinear degenerate problem arising in the theory of electrorheological fluids, Proc. R. Soc. A., 462 (2006), $2625-2641$

[12] M. Mihăilescu, Existence and multiplicity of solutions for a Neumann problem involving the $p(x)$-Laplace operator, Nonlinear Anal., 67 (2007), 1419-1425.

[13] B. Ricceri, On three critical points theorem, Arch. Math. (Basel), 75 (2000), 220-226.

[14] B. Ricceri, A general variational principle and some of its applications, J. Comput. Appl. Math., 113 (2000), 401-410.

[15] B. Ricceri, A further three critical points theorem, Nonlinear Anal., 71 (2009), 41514157.

[16] M. Růžička, Electrorheological Fluids: Modeling and Mathematical Theory, Lect. Notes Math., 1748. Berlin: Springer-Verlag, 2000.

[17] X. Shang and J. Zang, Three solutions for a pertubed Dirichlet boundary value problem involving the p-Laplacian, Nonlinear Anal., 72 (2010), 1417-1422.

[18] X. Shi and X. Ding, Existence and multiplicity of solutions for a general $p(x)$ Laplacian Neumann problem, Nonlinear Anal., 70 (2009), 3715-3720.

[19] D. Stancu-Dumitru, Multiplicity of solutions for anisotropic quasilinear elliptic equations with variable exponents, Bull. Belg. Math. Soc. - Simon Stevin 17 (2010) 875889.

[20] D. Stancu-Dumitru, Two nontrivial solutions for a class of anisotropic variable exponent problems, Taiwanese J. Math., 16 (4) (2012),1205-1219.

[21] D. Stancu-Dumitru, Multiplicity of solutions for a nonlinear degenerate problem in anisotropic variable exponent spaces, Bull. Malays. Math. Sci. Soc., 36 (1) (2013), $117-130$.

(Received: March 5, 2014)

(Revised: April 16, 2014)
Rabil Ayazoglu (Mashiyev)

Department of Mathematics Education Faculty of Education, Bayburt University 69000-Bayburt

Turkey

rabilmashiyev@gmail.com

Mustafa Avci

Faculty of Economics and Administrative

Sciences

Batman University

72000-Batman

Turkey 